\title{
A Discussion on RTN Variation Tolerant Guard Band Design Based on Approximation Models of Long-Tail Distributions for Nano-Scaled SRAM Screening Test
}

\author{
Worawit Somha and Hiroyuki Yamauchi
}

\begin{abstract}
This paper discusses, for the first time, how the guard band (GB) designs for the screening-test should be unprecedentedly changed when the shifting amounts of voltage-margin of the post-screening becomes larger than that of pre-screening. Three different types of amplitude-ratios of RTN to RDF (RTN/RDF: $0.25,1,4)$ are assumed in this discussion. The screening yield-loss impacts, made by: 1) larger ratio of RTN/RDF and 2) approximation-error of longer tailed RTN distribution, are discussed. It has been shown that the yield-loss may become crucial issues if RTN could not be reduced because the loss can become 5-orders of magnitude times larger than that for $40 \mathrm{~nm}$ when $R T N / R D F=1$. It has been found that the required accuracy-level of statistical model for approximating RTN tail-distributions significantly increases as RTN/RDF gets close to 1 . Intolerable yield-loss can be increased by 6-orders of magnitude due to its errors of the GB designs. A fitting method to approximate a longer tailed RTN Gamma-distribution by simple Gaussian mixtures model (GMM) is proposed. It has been verified that the proposed method can reduce the error of the fail-bit predictions by 2-orders of magnitude while reducing the iterations for EM step convergence to $1 / 16$ at the interest point of the fail probability of $10^{-12}$ which corresponds to the design target point to realize a 99.9\% yield of 1Gbit chips.
\end{abstract}

Index Terms-Mixtures of Gaussian, random telegraph noise, EM algorithm, heavy-tail distribution, long-tail distribution, fail-bit analysis, static random access memory, guard band design.

\section{INTRODUCTION}

The guard band (GB) design for Static Random Access Memory (SRAM) for the screening-test has become an unprecedentedly crucial challenge because the increased time-dependent (TD) margin variations-failure can't be screened by ordinary functional test any more resulting from: (1) significant increase in TD-margin variations after the screening, which will become much larger than non-TD variations before the screening (2) unprecedented rare-event (cdf of $10^{-12}$ ) to mimic a situation that defective cells are shipped to the market after the screening [1]-[3] as the bit density in an LSI chip continuously increases, and (3) more complex convolution models caused by the increased number of TD and non-TD heterogeneous random variables to predict the total yield-loss.

For example in (3), Vth distributions of nano-scaled CMOS have clearly shown that we have to consider not only

Manuscript received December 15, 2012; revised March 10, 2013.

W. Somha and H. Yamauchi are with the Information Intelligent System Fukuoka Institute of Technology, 3-30-1, Wajiro-Higashi, Higashi-ku, Fukuoka, Japan (e-mail: bd12002@ bene.fit.ac.jp, yamauchi@fit.ac.jp).
non-TD spatial random dopant fluctuation (RDF) but also TD temporal Vth variations due to Random Telegraph Noise (RTN) [4]-[5]. It has been well shown in the references [4]-[5] that distributions for Vth modulation $(\Delta \mathrm{Vth})$ amplitude due to RDF and RTN are obeyed to Gaussian and Gamma distributions, respectively. In addition, the increasing paces of $\Delta \mathrm{V}$ th amplitude are differently dependent on the MOSFET channel-size (LW) like the below expressions of (1) and (2).

$$
\begin{gathered}
\Delta \mathrm{Vth}(\mathrm{RDF}) \propto \mathrm{AVt}(\mathrm{RDF}) / \sqrt{\mathrm{LW}} \\
\Delta \mathrm{Vth}(\mathrm{RTN}) \propto \mathrm{AVt}(\mathrm{RTN}) / \mathrm{LW}
\end{gathered}
$$

where $\mathrm{AVt}(\mathrm{RDF})$ and $\mathrm{AVt}(\mathrm{RTN})$ are Pelgrom coefficients for RDF and RTN, respectively. Assuming the LW is scaled down to 0.5 every process generation, the $\Delta \mathrm{Vth}$ increasing paces of RTN is a $1.4 \mathrm{x}$ faster than that of RDF. This means that TD- $\Delta \mathrm{Vth}(\mathrm{RTN})$ will soon exceed non-TD- $\Delta \mathrm{Vth}(\mathrm{RDF})$ and becomes a dominant factor of whole margin variations. According to the references [4]-[5], there will come the time soon around $15 \mathrm{~nm}$ scaled CMOS era.

This indicates that unprecedented GB designs are needed to avoid the out of spec after the screening. Because the required GB voltages will be no longer small fraction of the whole margins, the conventional GB design criteria with the screening test won't be effective any more.

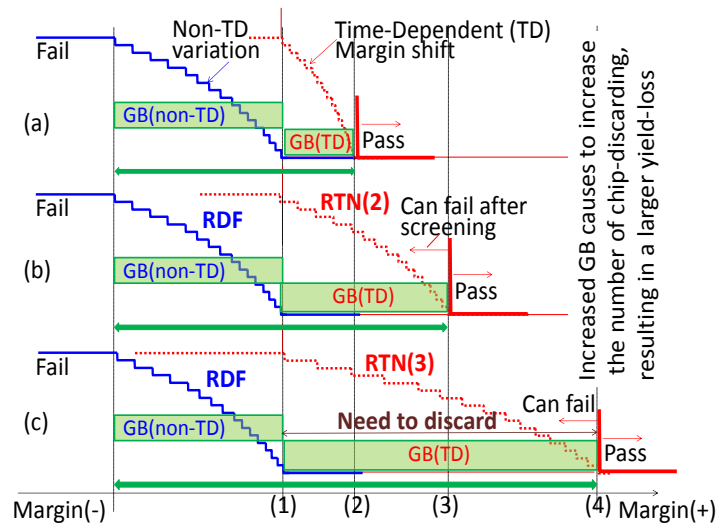

Fig. 1. Change of the GB ratio for non-TD to TD variations caused by RDF and RTN: (a) RTN<RDF, (b) RTN=RDF, (c) RTN $>$ RDF. Increased GB

causes to increase the number of discarding chip at screening test.

To make clear the issues we will discuss in this paper, the concepts of what will happen at that time are shown in Fig. 1 and Fig. 2. The GB (TD) in Fig. 1 refers to the GB voltages corresponding to the shifting amount of the margin variations due to RTN. As the GB(TD) for RTN required for avoiding the out of spec becomes larger with ever increased RTN and 
exceeds the GB(non-TD) for RDF, the number of discarding chips after the screening will be no longer neglected, as shown in Fig. 1.

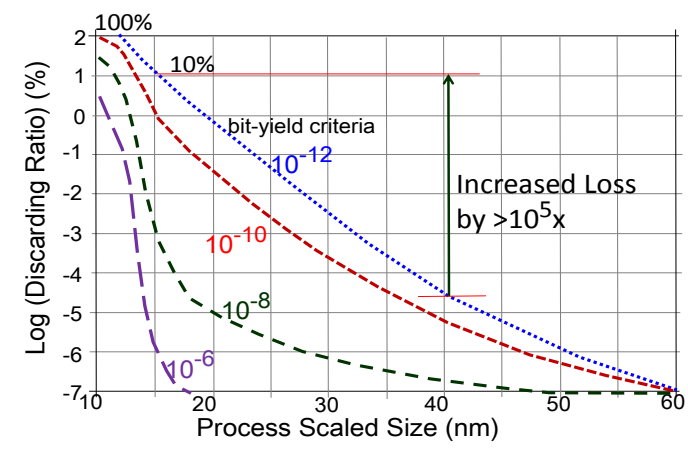

Fig. 2. Increased chip discarding ratio of $15 \mathrm{~nm}$ can be a $10^{5} \mathrm{x}$ larger than that of $40 \mathrm{~nm}$. Bit-yield criteria is defined as the required fail-probability, i.e., $1 \mathrm{e}-12$ is for $99.9 \%$ yield of $1 \mathrm{Gbit}$ chip.

Until $15 \mathrm{~nm}$, the percentage of the number of discarding chip required to avoid the out of spec after the screening can be increased by 5 orders of magnitude compared to that of 40nm, as shown in Fig. 2. It has been indicated that almost chips have to be discarded around $15 \mathrm{~nm}$ process generation to avoid the out of spec in the market after shipped, when considering its margin shifts by increased RTN tails of case-(c) of Fig. 1.

In order to discuss the impacts of rapidly increased TD-variations on the GB design for screening-test, 3 cases $(0.25,1,4)$ of variations-amplitude ratios of RTN/RDF are assumed in this discussion, as shown in Figs. 3-4.

Marked (1), (2), and (3) in Fig. 4 represent for the 3 cases of relationship between the RTN/RDF ratios of $0.25,1$, and 4 , respectively. Here, we also assumed the 3 cases for RDF: $\mathrm{RDF} 1, \mathrm{RDF} 2$, and RDF3. Its different trends also make impacts on the trend of RTN/RDF ratios. Since the advanced CMOS device tends to change to much less-dopant body devices like FinFET, ultra-thin body SOI, and nano-wire FET, RDF trends can be slow-down like the pace for RDF2 or RDF3, as shown in Fig. 4.

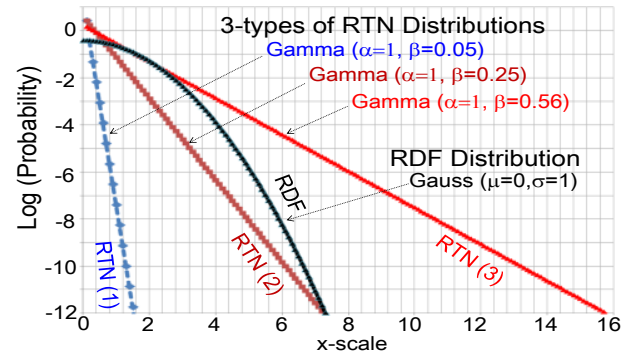

Fig. 3. Relationships between the distributions for RDF (Gauss) and three RTN (1), RTN(2), and RTN(3) (Gamma of $\beta=0.05,0.25$, and 0.56, respectively).

The yield-loss impacts made by: 1) the ratio of RTN/RDF and 2) approximation-errors of non-Gaussian RTN distribution by various statistical models are discussed in the following sections.

Here is how the rest of this paper is organized. In Section II, we refer to some of the example as evidence indicating how the GB design for screening test will be changed when RTN becomes dominating over whole margin variations. In Section III, we will discuss and present our recursive algorithm for constructing approximating Gaussian mixtures model (GMM). In Section IV, we refer to some of the example as evidence indicating if the proposed models can approximate well the heavy long-tailed distributions. We give a precise fail-bit count prediction. We rigorously prove that it is possible to approximate many long-tailed distributions by mixtures of Gaussian distributions. Finally, we state our conclusion in Section V.

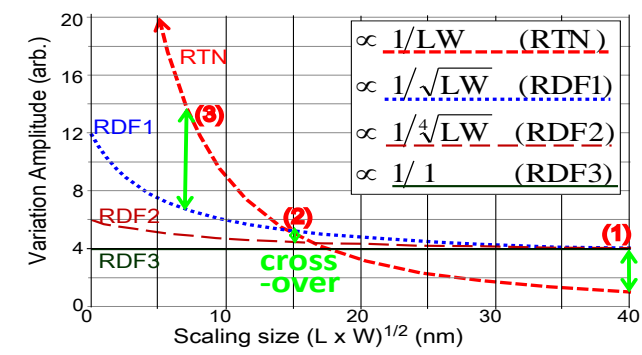

Fig. 4. Trends of variation amplitude of RTN and RDF.

The increasing pace of RTN is assumed as proportional to $1 / \mathrm{LW}$. Three cases of increasing pace of RDF are assumed: RDF1, 2 and 3, making impact on RTN/RDF.

\section{DisCUSSIONS ON SCREENINGS BASED ON EXAMPLES}

The examples of the effects of tail distributions on the shifted screening points are given in Figs. 5-7 for different RTN amplitudes of RTN(1), RTN(2), and RTN(3), respectively.

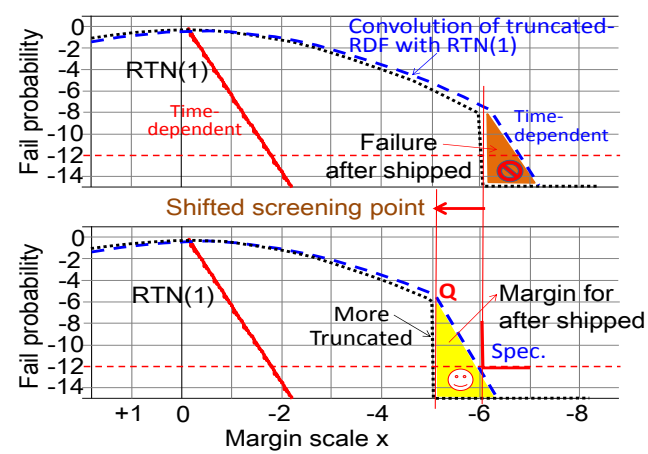

Fig. 5. RTN(1) impacts on tails after screening. To avoid any fail after shipped, screening point has to be shifted by 1 of $\mathrm{x}$. This causes additional chip discarding.

The tails of non-TD-margin distributions by RDF are truncated by screening and additional tails are added after screening by RTN effects, as shown in Figs. 5-7. The convolution of the two distributions of truncated RDF and RTN predicts the changes of whole margin distributions in 10 -years by RTN after screening.

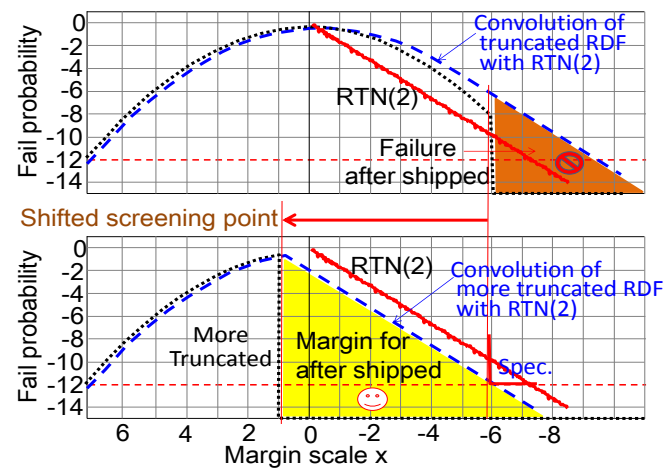

Fig. 6. RTN (2) impacts on tails after screening. To avoid any fail after shipped, screening point has to be shifted by 7 of $x$. This results in additional chip discarding. 


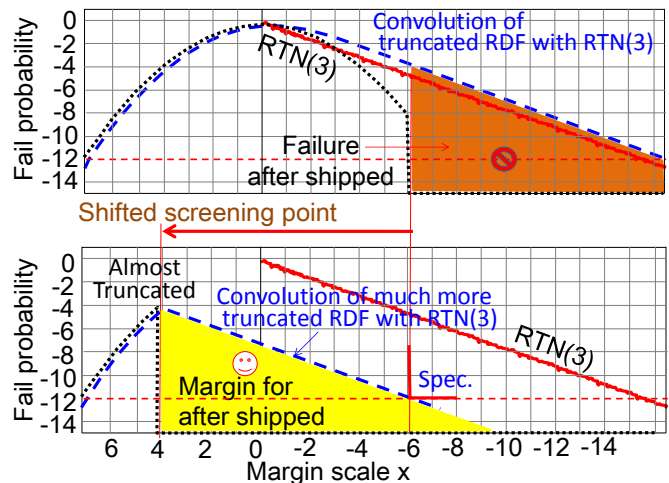

Fig. 7. RTN (3) impacts on tails after screening. To avoid any fail after shipped, screening point has to be shifted by 10 of $\mathrm{x}$. This discards almost of the chips before shipped.

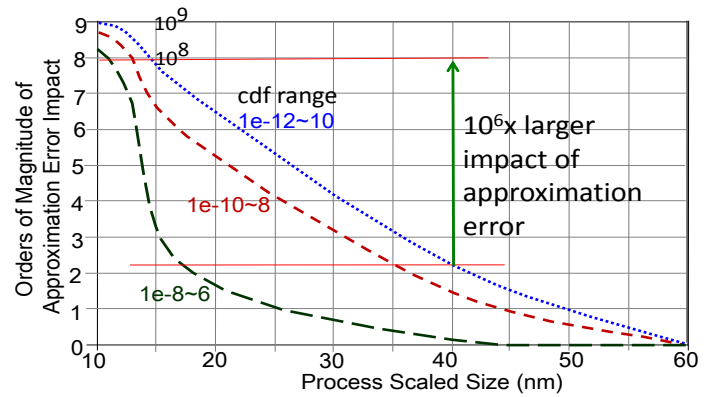

Fig. 8. Increased impact of approximation error on the trouble of excessive under-estimation/over- estimation of the yield.

As can be seen in the Fig. 5-Fig. 7, the shallower-angled slope of RTN distribution makes the length of tail longer and heavier. It is shown that a longer tail makes the screening point more shifted, e.g., $\Delta x=1,7$, and 10 are shifted by RTN(1), RTN(2), and RTN(3), as shown in Figs. 5-7, respectively. In these examples, the screening point are assumed as $\mathrm{x}=6$, as can be seen by the truncated Gaussian distributions. It is worth mentioning that the impacts of truncated distributions on the convolution results depend on the RTN slope. If the slope is steeper than Gaussian like RTN (1), distribution of convolution has a folding point like Q shown in Fig. 5. In contrast, the convolution with RTN(2) and (3) does look like no effects on any truncated points. This is because the tails of those RTN are longer than that of Gaussian. It is worth noting that the tail of convolution is the same as one of RTN. This means that the reducing the error of approximations to RTN long-tail Gamma distribution is crucial challenge in the GB design for screening test. The effects of yield-loss by excessive chip-discarding made by the error of RTN approximation are shown in Fig. 8.

More rarely event-analysis like a smaller cdf $<10^{-12}$ requires a higher accuracy at a longer tail position (larger $\mathrm{x}$ ) and its required error level depends on the interest cdf values. Thus, its errors in the three ranges of $10^{-(12 \sim 10)}, 10^{-(10-8)}$, $10^{-(8 \sim 6)}$ are measured in this paper, as shown in Fig. 8. It is worth mentioning that the accuracy of the RTN approximation by the statistical model becomes more important as the process is scaled down. It stems from the increased ratio of RTN/RDF, causing larger and heavier RTN tail distributions due to the process scaling, as shown in Fig. 4. It has been found that the errors affecting the discarding chip counts at $15 \mathrm{~nm}$ can be over 6 -orders of magnitude larger than that at $40 \mathrm{~nm}$ in the range of $\mathrm{cdf}=10^{-(12 \sim 10)}$ as can be seen in Fig 8. (target of fail-bit criteria: $99.9 \%$ chip yield for
1Gbit 10Mbit chips)

As explained in this section, the accuracy of the approximation of RTN distributions is unprecedentedly crucial challenge for the screening test design to avoid an excessive under-estimation/over- estimation of the yield.

\section{Proposed Statistical Approximation Model for RTN GAMMA DISTRIBUTION}

One of the challenges we should address is how to reduce the approximation error in the tails for the different types of distributions of Gaussian of RDF, Gamma of RTN and its tails of convolution results of those.

The main contribution in this section is to point out that it is possible to approximate the long tailed distributions by mixtures of convenient Gaussian probability distributions, so that available yield-prediction models can be effectively analyzed and so that the effect of the long tailed distributions upon the fail-bit count accuracy can be analytically determined. This is because the convolution result of linear combinations of Gaussians becomes also Gaussians and can be expressed by the analytical expressions, which allows using error-function for estimating the error counts. This makes it easier to predict the fail-bit counts before and after screening at the stages of both circuit design and screening test.

Expectation-maximization (EM) algorithm [6], which is an iterative procedure that maximizes the likelihood of Gaussian mixtures models (GMM), is well known as easy and convenient means to approximate GMM to the non Gaussian distributions. However, this fitting algorithm tends to prioritize the non-tail region in which the sensitivity to increase the likelihood becomes much larger than that for the tail region as shown in Fig. 9.

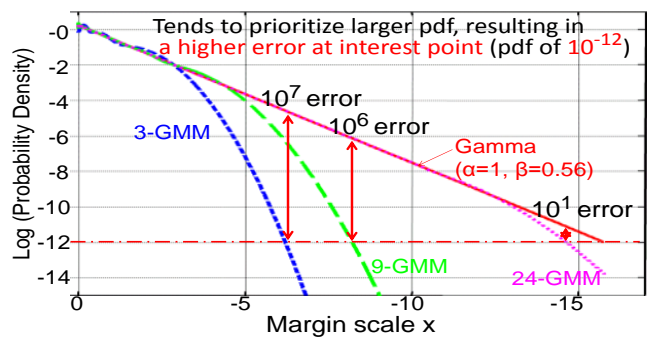

Fig. 9. Approximation error comparisons between 3, 9, 24-GMMs cases: errors of orders of $10^{7}, 10^{6}$, and $10^{1}$, respectively

Since the interest region for analyzing the fail-bit counts in the rare events is in the tail region (at probability of $10^{-12}$ ), the EM algorithm for this application leads to a significant fail-bit count error of orders of $10^{7}$, as shown in Fig. 9. Even if increasing the number of GMM from 3 to 9 and 24, the significant error of orders of $10^{6}$ and $10^{1}$, respectively, are still remained, as shown in Fig. 9. In almost all fail-bit analyses, the distribution of interest only matters in the tail-region of probability of orders of $10^{-12}$ [1]-[3]. Thus, this is a crucial challenge we should address until the time comes for the rare-event SRAM yield predictions.

In order to solve these crucial issues, we develop a remarkably simple EM-based fitting algorithm, which is based on: 1) adaptive partitioning of the long tailed distributions such that the log-likelihood of GMM is maximized in each segmentation and 2) copy and paste 
fashion with an adequate weight into each partition for constructing the whole long-tail distributions.

The concepts of the proposed EM-based approximation are shown in Fig. 10 and algorithm is described from step-(1) to step-(4).

1) $1^{\text {st }}$-step is to do approximation by $3-G M M$ between $\mathrm{X} 0$ and X3. And find the point of X1, where likelihood of 3-GMM is maximized.

2) $2^{\text {nd }}$-step is to do the same thing as (1) between $X 1$ and $X 3$. And find the point of $\mathrm{X} 2$, where likelihood of 3-GMM is maximized.

3) $\quad 3^{\text {rd }}$-step is to do the same thing as (2) between $X 2$ and $X 3$. And find the point of $\mathrm{Xn}$, where likelihood of 3-GMM is maximized between $\mathrm{X} 2$ and $\mathrm{X} 3$.

This flow can be repeated until the likelihood of whole GMM can be maximized.

Suppose here that we try to approximate a given Gamma distribution of ( $\alpha=1, \beta=0.56$ ) by using 3-GMM and 9-GMM. The examples of approximations of the segmentations of $(\mathrm{x}=0 \sim 0.38)$ by $3-\mathrm{GMM}$ and $(\mathrm{x}=0 \sim 1.4)$ by 9-GMM, respectively, are shown in Fig. 11.

It is worth mentioning that the approximation by 9-GMM looks much more prioritizing the non-interest area of $(\mathrm{x}=0 \sim 0.2)$ over $(\mathrm{x}=1 \sim 1.4)$. This causes a larger error in the tail region of $(x=1 \sim 1.4)$ than that of $(x=0 \sim 0.2)$. Since the width between $\mathrm{X} 0$ and $\mathrm{X} 1$ are roughly proportional to the number of GMM, the 9-GMM can lead a larger error than 3-GMM. This algorithm can allow approximating any heavy-long tailed distributions by convenient short-tail Gaussian probability distributions. This allows us to use only Gaussian distributions when doing convolution of Gaussian-RDF and Non-Gaussian-RTN distributions. The convolution results can be given by analytical simple and convenient expressions of just linear combination of Gaussian, which can give us the fail-bit count by just summing up the values of error-functions for each Gaussian of whole GMM.

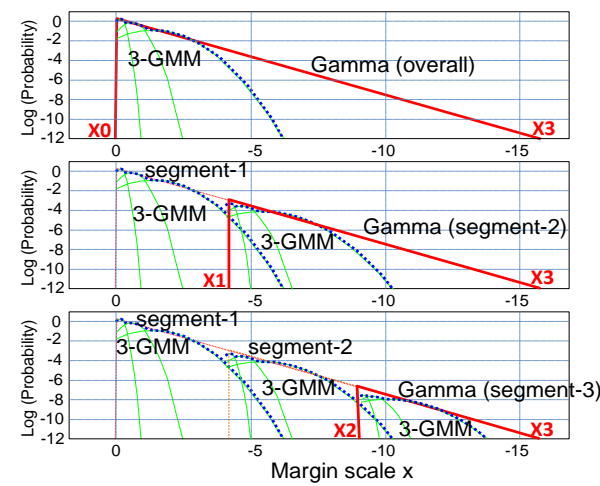

Fig. 10. Concepts of the proposed approximation algorithm. X1 and X2 are decided such that likelifood of each 3-GMM can be maximized.

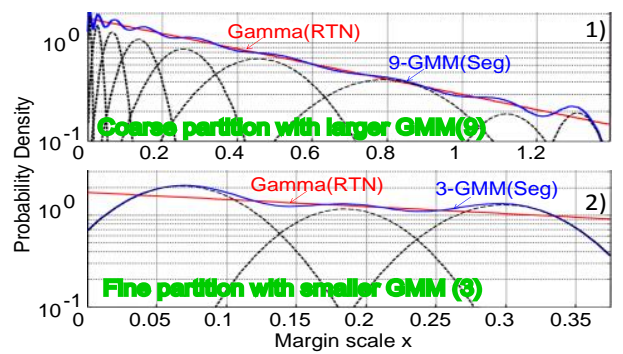

Fig. 11. Comparisons of GMM approximation to Gamma distribution between 1) coarse partitioning with 9-GMM and 2) fine partition with 3-GMM

\section{DisCUSSION ON ACCURACY OF STATISTICAL APPROXIMATION MODEL FOR RTN DISTRIBUTION}

To illustrate the effects of the proposed scheme on the reduction of the approximation-error in the long tails, the following examples are assumed: (1) ratio of how fast does the tail decay of Gaussian-RDF and Gamma-RTN, i.e., its parameters are assumed as follows: $(\sigma=1, \mu=0)$ for Gaussian, ( $\alpha=1, \beta=0.56$ ) for Gamma. The relationship between the two distributions and its convolution are shown in Figs. 5-7 and (2) comparisons of the following 6 approximation-models of Gamma distribution ( $\alpha=1, \beta=0.56)$ : (a) the number of 3, 9, 24, and 128 of GMMs are used for fitting the whole distribution (no segmentation) and (b) the number of 3 and 9 of GMMs are used for approximating each segmentation comprising whole distribution.

Fig. 12 shows the comparisons of the convolution results between the cases with and without segmentation schemes. It is found that 3-GMM segmentation scheme can reduce the errors by 7-orders and 4-orders of magnitude at the fail probability of $10^{12}$, as shown in Fig. 12. It is worth mentioning that 3-GMM segmentation scheme provides a better approximation than the case of 24-GMM, as shown in Fig. 12. In order to characterize the error of each convolution result, the "golden", which is given by the numerical calculation of convolution of Gaussian and Gamma distributions, is used as a reference. The numbers of fail-bit count errors for each approximation model are compared, as shown in Fig. 13. The number of fail-bit count error is defined as the difference in the cdf value between the "golden" and that for each model.

It is worth mentioning that the relationship of "which is better" is dependent on the X-scale, as shown in Fig. 13. For example, 128-GMM is the best in $\mathrm{X}=(-6 \sim-12)$ in which the fail-probability is larger than $10^{-8}$ (shown in Fig. 13). In contrast, the proposed one can reduce the error best in $\mathrm{X}=$ $(-12 \sim-16)$ in which the fail-probability is smaller than $10^{-8}$ where there is the most interest point for the GB designs.

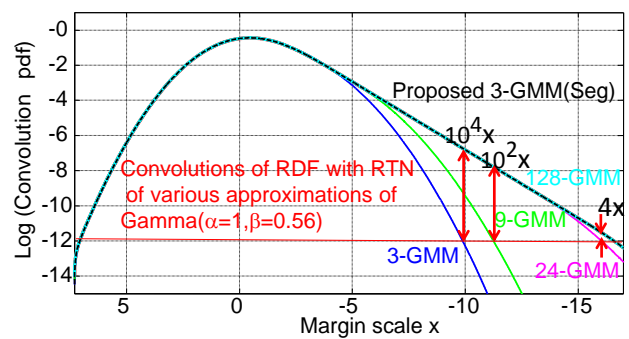

Fig. 12. Comparisons of the convolution results between the cases with and without segmentation schemes of 3, 9, 24, and 128-GMMs. Proposed one can reduce the error by $10^{4} \mathrm{x}, 10^{2} \mathrm{x}$, and $4 \mathrm{x}$ than 3 , 9, and 24-GMMs, respectively

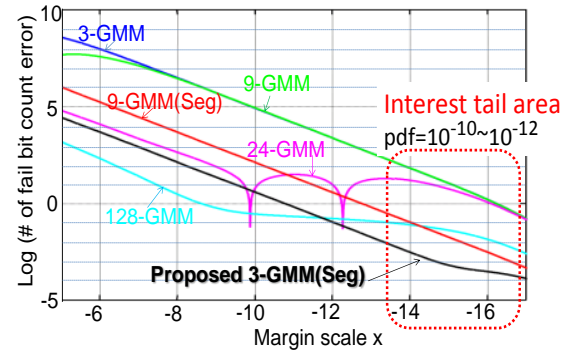

Fig. 13. Comparisons of the numbers of fail-bit count errors between 6 approximation means of 3, 9, 24, and $128 \mathrm{GMM}$ without segmentation and 3 and 9-GMM with segmentation schmes. 
When discussing the screening conditions for volume production, the expected yield-loss should be assumed. We assumed the target here that fail-probability is $10^{-12}$ to realize $99.9 \%$ yield of $1 \mathrm{Gbit}$ memory chip, which is a quite realistic target. Thus, our most interest point of $\mathrm{x}$ is around $\mathrm{x}=-16$, where fail-probability is around $10^{-12}$, as you can see by Fig. 12. In that sense, we can say that our proposed 3-GMM segmentation method can provide the best approximation compared with others.

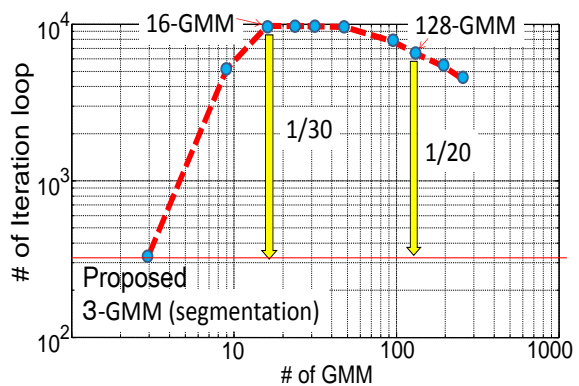

Fig. 14. The number of iterations of EM steps are compared between 3-GMM segmentation scheme and 128-GMM w/o segmentation. The required iterations of EM steps are reduced by $1 / 30$ and 1/16, respectively, compared with 16-GMM and 128-GMM w/o using segmentation scheme.

As mentioned earlier, 9-GMM segmentation is worth than 3-GMM segmentation in the wide range of $x=-6 \sim-16$ because the variation of probability becomes larger and the density of GMM in area of lower probability becomes much smaller as shown in Fig. 13. Regarding the cases without using segmentation, the number of errors is $1 \sim 4$ orders of magnitude larger than that for the proposed 3-GMM segmentation scheme.

Since the reduction of the required iterations for EM step convergence is also crucial in practical application, the relationship between the number of GMM and the number of iterations are compared, as shown in Fig. 14. It is worth noting that the proposed 3-GMM segmentation scheme can reduce not only the error but also the EM-step iterations. The 3-GMM segmentation scheme can reduce by to $1 / 30$ and $1 / 20$ of that for 16 and 128-GMM without segmentation scheme.

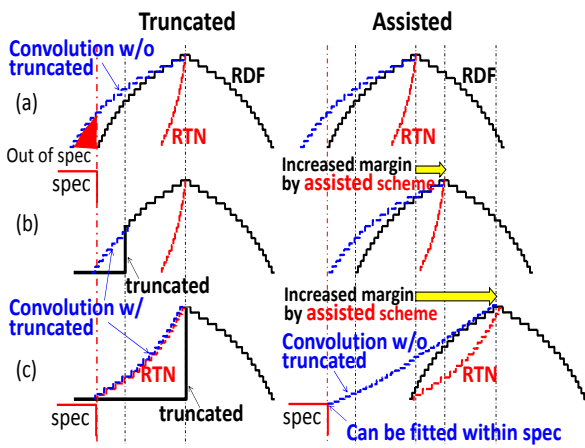

Fig. 15. Comparisons of GB designs to avoid any out of specs after screening: (left) pre-truncating less margin chips so that out of spec can't happen with margin-shift by RTN and (right) increasing margin by using the assist circuit [1] such that convolution results can be fitted within spec.

\section{ASSISTED MARGIN SHIFTS}

There are two potential means to avoid any out of spec after screening, as shown in Fig. 15: (1) pre-truncating the less-margin chips so that "out of spec" never happens with the RTN-caused margin shifts and (2) increasing the margin by using the assist circuits [1]-[3] so that the convolution results of RTN and RDF can be fit within the spec.

As can be seen in Fig. 5-Fig. 8, the yield-loss will become prominent by truncated chips. To address this challenge, we will need to design the margin assist circuits for RTN-caused variations as shown in Fig. 15, which are conventionally used for non-TD GB designs for RDF-caused variations [1]-[3]. In this symposium, more detailed discussions on the above concepts will be given.

\section{CONCLUSION}

In this paper, we have discussed, for the first time, how the GB design should be changed when the shifting amounts of operating voltage margins after screening can be unprecedentedly larger than that of before screening. It has been found that yield-loss (chip-discarding) by screening test may become crucial issues if RTN could not be reduced or eliminated because it can be about 5-orders of magnitude times larger than $40 \mathrm{~nm}$ when $\mathrm{RTN} / \mathrm{RDF}=1$. It has been pointed out that intolerable yield-loss by wrong GB design can be increased by 6-orders of magnitude and the required accuracy of statistical model for approximating the tails of RTN distributions will become unprecedentedly crucial as the process is scaled down and body doping becomes less than that for RDF in coming process generation like $15 \mathrm{~nm}$ and beyond.

In this paper, we have also developed an effective simple algorithm for approximating the tails of RTN distributions by convenient and simple GMM even if its tail becomes heavier and longer. It is verified that the proposed method can reduce the error of the fail-bit predictions by 2-orders of magnitude while reducing the iterations for EM step convergence to 1/16 at the interest point of the fail probability of $10^{-12}$ which corresponds to the design point to realize a $99.9 \%$ yield of 1 Gbit chips.

We have pointed out that the proposed scheme is a candidate fitting algorithm for the distributions of the future SRAM operating margins, which will be crucial not only for the circuit design but also the GB design for screening test when RTN variables becomes larger than that of RDF.

\section{ACKNOWLEDGMENT}

The authors are grateful to Yan Zhang, Yu Ma for their helps.

\section{REFERENCES}

[1] H. Yamauchi, "A Discussion on SRAM Circuit Design Trend in Deeper Nanometer-Scale Technologies," IEEE Transactions on Very Large Scale Integration Systems, vol. 18, no. 5, pp. 763-774, 2010.

[2] H. Yamauchi, "Embedded SRAM trend in nano-scale CMOS," IEEE International Workshop on Memory Technology, Design and Testing, 2007, pp. 19-22.

[3] H. Yamauchi and Tutorials, "Variation tolerant SRAM circuit design," in IEEE ISSCC 2009 and IEEE A-SSCC 2008.

[4] K. Takeuchi et al., "Comprehensive SRAM design methodology for RTN reliability," Digest of IEEE Symposium on VLSI Technology, 2011, pp. 130-131.

[5] K. Takeuchi et al., "Direct observation of rtn-induced sram failure by accelerated testing and its application to product reliability assessment," Digest of IEEE Symposium on VLSI Technology, 2010, pp. 189-190

[6] T. K. Moon, "The expectation-maximization algorithm," Signal Processing Magazine, IEEE, vol. 13, no. 6, pp. 47-60, 1996. 


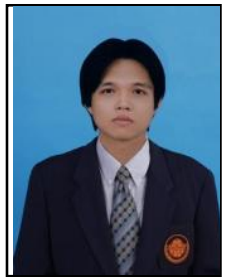

Worawit Somha received master degree in electrical engineering from King Mongkut's Institute of Technology Ladkrabang (KMITL), Bangkok, Thailand. His master thesis was on "Vector Quantizers for Speech Coding and there Implementation on TMS-320C30". Since 1995 he has given a lecture for bachelor degree student in subject of "Introduction to Digital Signal Processing" at KMITL as an assistance professor, and his research area is speech coding. Since 1997 he has worked with the company in the position of consulting engineering.

Since 2012 he has got the scholarship from KMITL for D. Eng. student program and now being pursuing $\mathrm{PhD}$ degree in major of intelligence information system engineering at Fukuoka Institute of Technology.

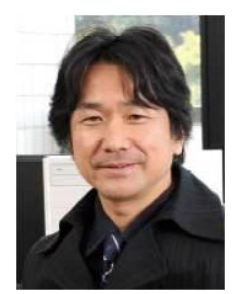

Hiroyuki Yamauchi received the Ph.D. degree in engineering from Kyushu University, Fukuoka, Japan, in 1997. His doctoral dissertation was on "Low Power Technologies for Battery-Operated Semiconductor Random Access Memories". In 1985 he joined the Semiconductor Research Center, Panasonic, Osaka, Japan. From 1985 to 1987 he had worked on the research of the submicron MOS FET model-parameter extraction for the circuit simulation and the research of the sensitivity of the scaled sense amplifier for ultrahigh-density DRAM's which was presented at the 1989 Symposium on VLSI Circuits. Dr. Yamauchi received the 1996 Remarkable Invention Award from Science and Technology Agency of Japanese government and the highest ISOCC2008 Best Paper Award. He had been serving a program committee of ISSCC for long periods, from 2002 through 2009.

He served a program committee of IEEE Symposium on VLSI Circuits from 1998 through 2000 and has come back and been serving again since 2008. He is also serving A-SSCC since 2008. 